\title{
Curcumin combined with low-intensity ultrasound suppresses the growth of glioma cells via inhibition of the AKT pathway
}

\author{
Guohong SHI, Zhen ZHANG*, Yi FANG, Donglin BIAN, Zhiqun BAI \\ Department of Ultrasonic Diagnosis, The First Affiliated Hospital, China Medical University, Shenyang, Liaoning, China \\ ${ }^{*}$ Correspondence: $2825166781 @ q q . c o m$
}

Received June 5/ Accepted October 27, 2020

\begin{abstract}
Malignant glioma is the most lethal form of brain cancer, and effective therapeutic modalities remain unavailable to date. We aim to investigate whether low-dose curcumin combined with low-intensity ultrasound (LIUS) effectively suppresses the growth of glioma cells and elucidate the underlying mechanisms. Glioma cells were treated with LIUS and curcumin. Subsequently, the effects of LIUS and curcumin on glioma cells were determined by CCK-8 assay, EdU assay, and flow cytometry analysis, respectively. Western blot analysis was performed to examine the levels of apoptosis-associated proteins and the proteins related to the AKT pathway. The proliferation assay showed that combined treatment with LIUS and curcumin synergistically decreased proliferation in glioma cells. And cell apoptosis was promoted after LIUS-curcumin combination treatment, characterized by the occurrence of more apoptotic cells and a significant increase in Bax level and attenuated Bcl-2 expression. Moreover, the role of LIUS-curcumin combination in downregulation of the AKT pathway was observed. The AKT pathway activator SC79 reversed apoptosis and anti-proliferation induced by combined treatment with LIUS and curcumin. Our findings show that LIUS in combination with low-dose curcumin synergistically suppresses the growth of glioma cells via inhibition of the AKT pathway. LIUS plus curcumin may be a promising therapeutic strategy for preventing glioma growth.
\end{abstract}

Key words: low-intensity ultrasound, curcumin, glioma, AKT

Glioma is one of the most common types of malignant brain tumors, with high morbidity and mortality [1]. Combination therapy with temozolomide and radiotherapy, which is the standard of care for glioma, has increased the median overall survival by only 15-20 months [2]. Therefore, the development of novel therapeutic modalities for glioma is urgently needed.

Wang et al. reported that curcumin exerted suppressive effects against the growth of glioma cells [3]. Extensive studies have demonstrated that the anticancer effects of curcumin were primarily attributable to the induction of apoptosis and cell cycle arrest via targeting multiple signaling pathways [4, 5]. However, the poor solubility and limited bioavailability of curcumin alone limit its application in preventing glioma growth [6]. To enhance the therapeutic effect of curcumin and limit the side effects due to higher dosages, the concomitant use of another therapy is proposed.

Our previous publication showed that low-intensity ultrasound (LIUS) promoted glioma cell apoptosis [7]. Furthermore, the ultrasound is known to transiently increase the permeability of biological barriers, such as cell membranes and blood vessels, thereby increasing the intracellular uptake of drugs $[8,9]$.

The phosphoinositide 3-kinase (PI3K)/AKT/mTOR signaling pathway is one of the most frequently activated pathways in various human tumors, exhibiting the major survival pathway and promoting tumorigenesis [10-14]. And this pathway modulates multiple oncogenic processes, such as cell proliferation, angiogenesis, differentiation, apoptosis, and autophagy [15]. Therefore, inhibition of the AKT pathway may be a potential treatment option against human glioma [16]. In glioma cells, anti-tumor effects and suppression of the PI3K/AKT pathway were observed when using curcumin treatment $[17,18]$. However, due to the solubility and bioavailability issues of curcumin, it is difficult to deliver sufficient levels of curcumin to tumor cells. LIUS has been proved to promote the delivery of cytotoxic curcumin to cells in oral carcinoma [19].

In accordance with these findings, we hypothesized that combined treatment with low-dose curcumin and LIUS 
would achieve synergistic anti-tumor effects. Furthermore, we postulated that this effect is likely achieved via inhibition of the AKT pathway.

\section{Materials and methods}

Cell line culture. Human glioma cell lines U87 (ATCC) (glioblastoma of unknown origin) and U251 (RIKEN) (astrocytoma) were purchased from the Cell Bank of the Chinese Academy of Sciences (Shanghai, China). All cells were cultured in Dulbecco's modified Eagle's medium (DMEM, HyClone, USA), supplemented with $10 \%$ fetal bovine serum (FBS, HyClone, USA) and $1 \%$ penicillin-streptomycin in a humidified atmosphere of $5 \% \mathrm{CO}_{2}$ at $37^{\circ} \mathrm{C}$.

Ultrasonic exposure and grouping. The EMS-9 ultrasound generator (Shenzhen Delica Company, China) used in this experiment was operated at a frequency of $2 \mathrm{MHz}$. We sonicated each sample separately by moving the plate to the target of LIUS. First, we utilized a variety of ultrasonic intensities, and groups were divided into 50.4, 83.4, 142.0, 290.0 , and $474.0 \mathrm{~mW} / \mathrm{cm}^{2}$, respectively. Then, curcumin was dissolved in dimethyl sulfoxide (DMSO) to produce a $50 \mathrm{mM}$ stock solution. The concentrations of curcumin were set as follows: 5, 10, 15, and $20 \mu \mathrm{M}$. Curcumin (458-37-7) and the AKT pathway activator SC79 (305834-79-1) were purchased from Sigma-Aldrich (St. Louis, MO, USA).

To explore the effects of combined treatment with LIUS and curcumin on glioma cells, the following groups were defined: 1) untreated control group; 2) LIUS; 3) curcumin; and 4) the combination of curcumin and LIUS (curcumin + LIUS). Glioma cells in curcumin and curcumin + LIUS groups were cultured with curcumin $(10 \mu \mathrm{M})$ in the dark. Cells in the LIUS and curcumin + LIUS groups were subject to ultrasonic exposure $\left(83.4 \mathrm{~mW} / \mathrm{cm}^{2}\right)$ for $60 \mathrm{~s}$ in the dark. After $24 \mathrm{~h}$ of incubation, these cells were used for further experiments.

To further determine combined treatment with LIUS and curcumin-induced anti-cancer effects via inhibition of the AKT pathway, we employed the AKT pathway activator SC79. Treated glioma cells were divided into four groups: control group, SC79 group, curcumin + LIUS group, and curcumin + LIUS + SC79 group.

Cell counting kit-8 (CCK-8) assay. Cell viability was measured using the Cell counting kit-8 (CCK-8; Dojindo Molecular Technologies, Gaithersburg, USA) based on the manufacturer's indication. Briefly, glioma cells were planted into 96-well plates and cultured with different treatments for $24 \mathrm{~h}$. Then, $10 \mu \mathrm{l}$ CCK- 8 test solution was added to each well, and the plates were incubated for $2 \mathrm{~h}$ at $37^{\circ} \mathrm{C}$. Absorbance values were measured at $450 \mathrm{~nm}$ using a microplate reader (Thermo Fisher Scientific, Inc., USA).

5-Ethynyl-2'-deoxyuridine (EdU) assay. U87 and U251 cells were planted into 96-well plates and incubated for $24 \mathrm{~h}$ after different treatments. The Cell Light EdU DNA imaging kit obtained from RiboBio (Guangzhou, China) was used to determine cell proliferation according to the manufacturer's guide. Subsequently, the nuclei could be observed using an inverted fluorescence microscope (Nikon, Japan). The quantitative data were presented as the percentage of EdU-positive nuclei relative to the total number of nuclei counted.

Flow cytometry analysis. Apoptosis was evaluated with the Annexin V-FITC Apoptosis Detection kit (BD Biosciences, USA). Glioma cells $\left(2 \times 10^{5}\right.$ cells/well $)$ were seeded into 6-well plates and subjected to different treatments. After $24 \mathrm{~h}$, the cells were harvested and washed with PBS, and resuspended in $100 \mu \mathrm{l}$ of $1 \times$ binding buffer, followed by the addition of $5 \mu \mathrm{l}$ of propidium iodide (PI) and $5 \mu \mathrm{l}$ of Annexin $\mathrm{V}$-FITC to the cell suspension. The cells were incubated for $15 \mathrm{~min}$ in the dark at room temperature. Then, $400 \mu \mathrm{l}$ of $1 \times$ binding buffer was added to each tube. Flow cytometry was carried out to evaluate cell apoptosis using a FACSAria Flow Cytometer (BD, USA).

Western blot analysis. Glioma cells after different treatments were lysed in ice-cold RIPA buffer (Beyotime, Shanghai, China) for 15 minutes. Total protein concentrations were determined by a BCA kit (Beyotime, Shanghai, China). Afterward, protein samples were separated by sodium dodecyl sulfate-polyacrylamide gel electrophoresis (SDS-PAGE) and then transferred onto a polyvinylidene difluoride membrane using wet transfer at $80 \mathrm{~V}$ for $60 \mathrm{~min}$, and $120 \mathrm{~V}$ for $60 \mathrm{~min}$. Nonspecific binding sites were blocked with $5 \%$ skim milk powder at room temperature for $2 \mathrm{~h}$ and the membranes were incubated with primary antibodies as follows: Bax (1:2000, ab32503) and Bcl-2 (1:1000, ab196495) were purchased from Abcam (Cambridge, UK). AKT (1:1000, \#9272) and p-AKT Ser473 (1:1000, \#4060) were acquired from Cell Signaling Technology (Danvers, Mass). Secondary antibody (1:2000, SA00001-2) and anti-GAPDH antibody (1:10000, 10494-1-AP) were purchased from Proteintech (Chicago, IL, USA). An Image Lab software was employed to detect the protein bands and proteins were visualized using an enhanced chemiluminescent substrate (Thermo Fisher Scientific, Inc.).

Statistical analysis. All experiments were independently repeated at least three times. Values were analyzed with SPSS 19.0 software (IBM Corp., Armonk, NY, USA) or GraphPad Prism 6.01 software (La Jolla, CA, USA) and presented as mean \pm standard deviations (SD). Continuous data were analyzed with a one-way ANOVA followed by Tukey's post hoc test for multiple group comparison. A p-value $<0.05$ was considered as significant.

\section{Results}

Effects of LIUS and curcumin on glioma cell viability. To determine the effect of various ultrasonic intensities $\left(50.4,83.4,142.0,290.0\right.$, and $\left.474.0 \mathrm{~mW} / \mathrm{cm}^{2}\right)$ on glioma cell viability, CCK-8 assay was carried out after sonication. The viability rates of glioma cells showed a reducing tendency with elevated ultrasonic intensity (Figure 1A). The results 
demonstrated that LIUS suppressed the growth of glioma cells in an intensity-dependent manner, but that there was no obvious effect when the sonication intensity was below $83.4 \mathrm{~mW} / \mathrm{cm}^{2}$.
To evaluate the effect of different concentrations $(5,10$, 15 , and $20 \mu \mathrm{M}$ ) of curcumin on cell viability, U87 and U251 cell viability was measured by CCK- 8 assay after incubation with curcumin for $24 \mathrm{~h}$. There was no significant difference
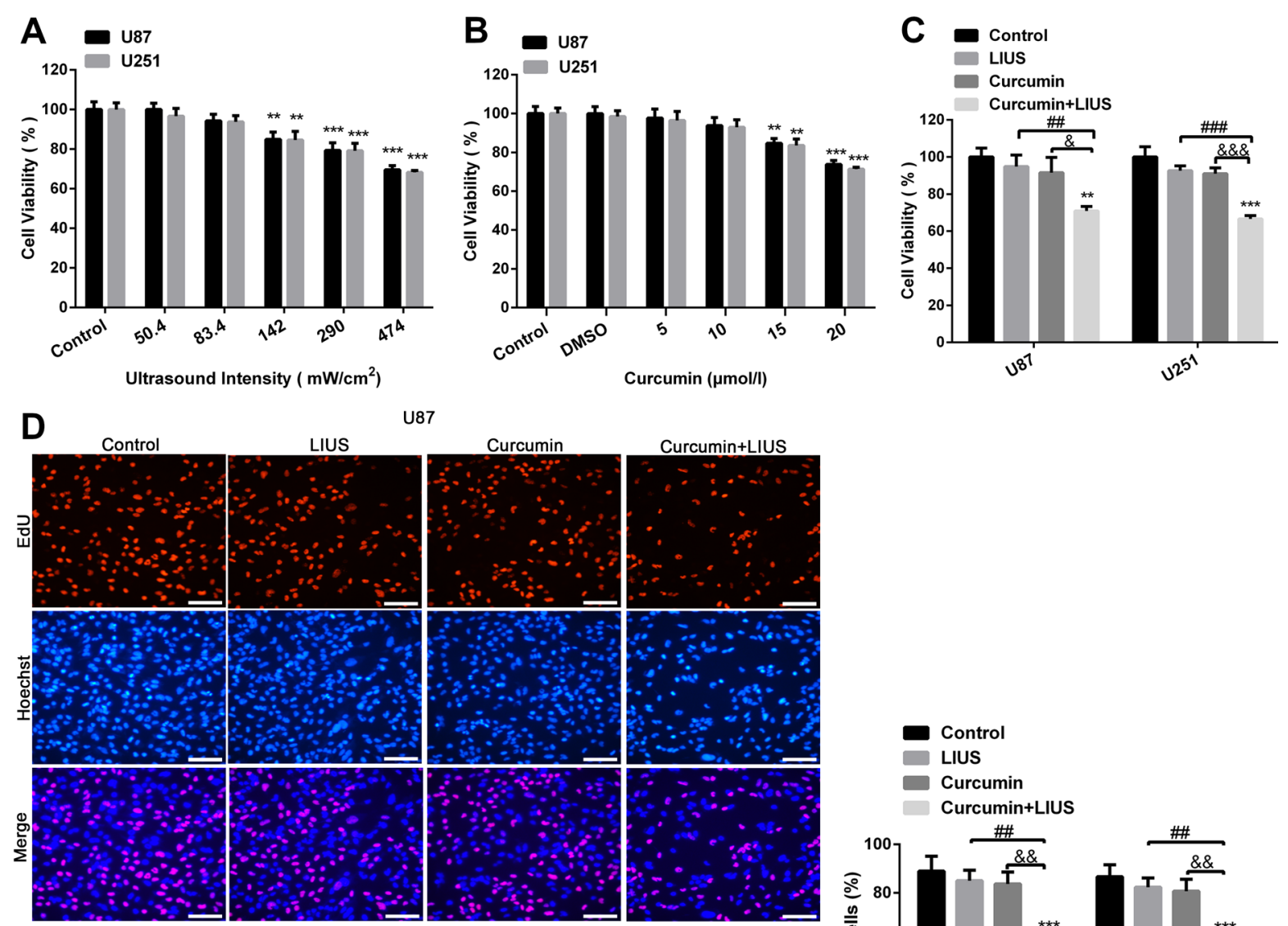

U87

Curcumin $(\mu \mathrm{mol} / \mathrm{l})$
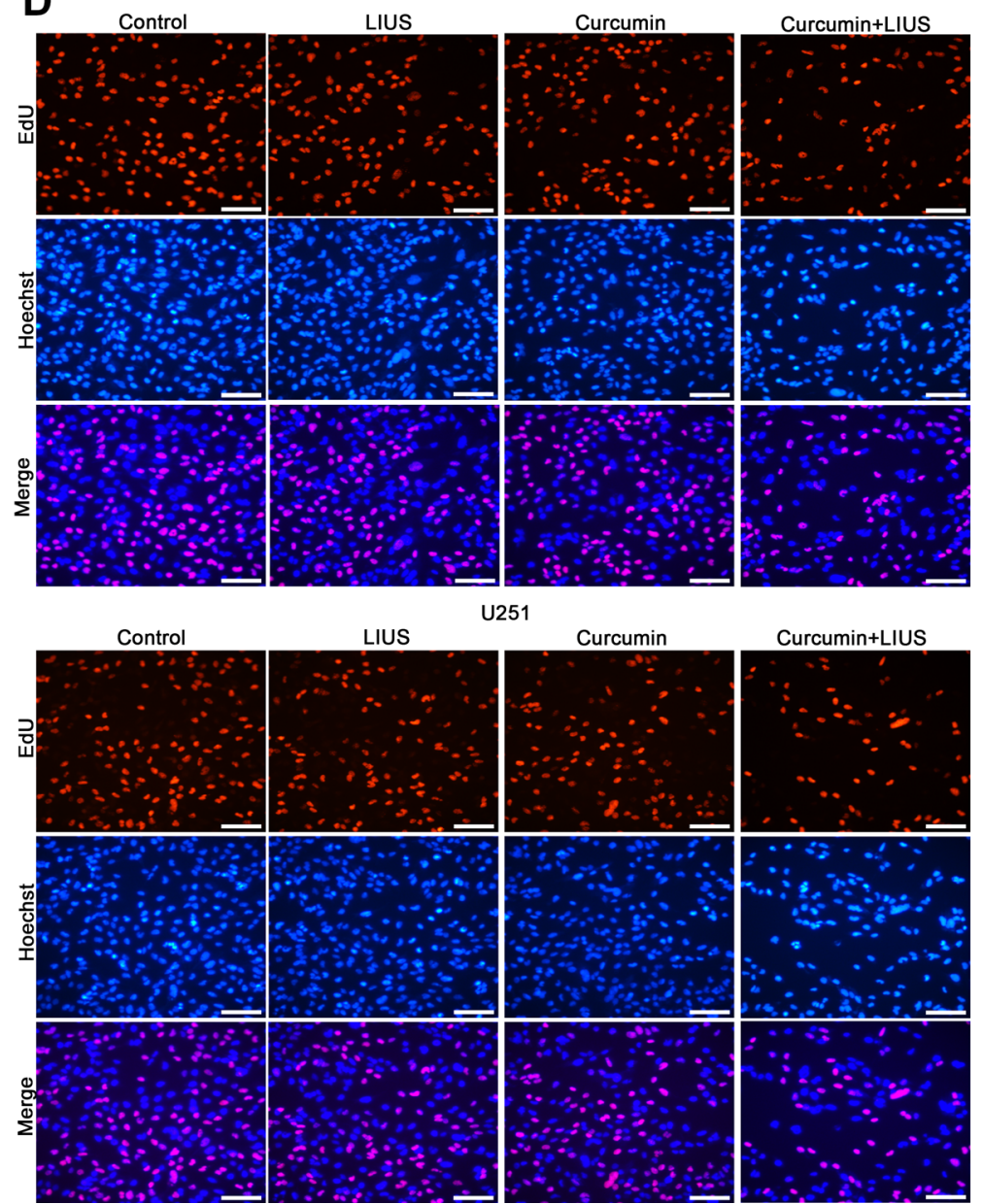

Curcumin

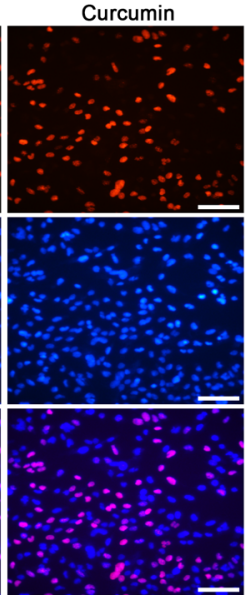

Curcumin+LIUS
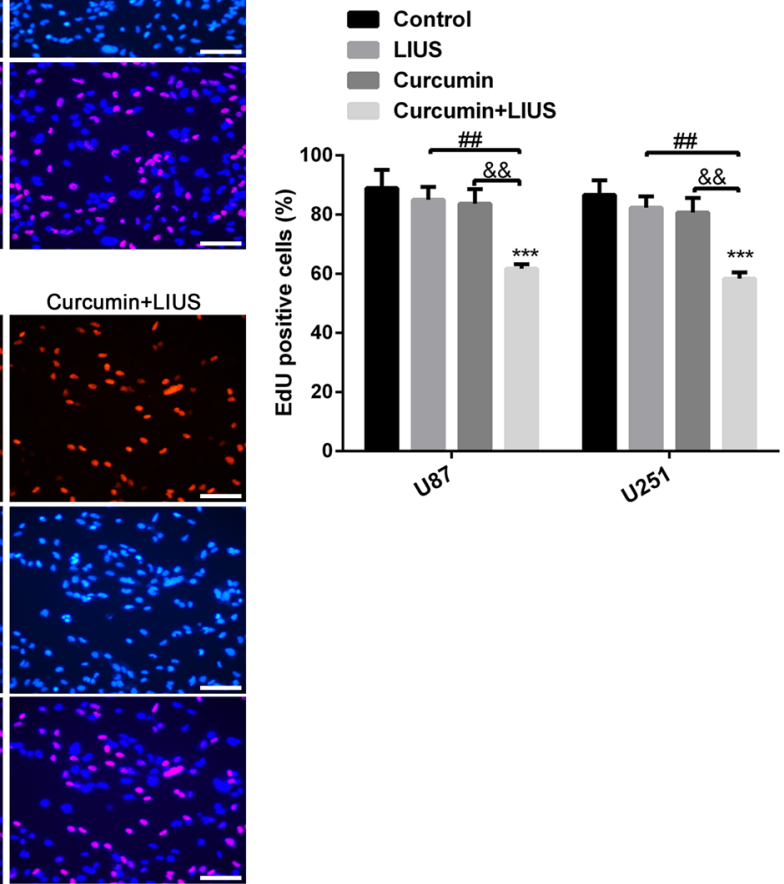

Figure 1. The effects of LIUS and curcumin on the proliferation of glioma cells as analyzed by CCK- 8 assay and EdU assay. A) The effect of varying ultrasonic intensities on the growth of glioma cells. B) The effect of various curcumin concentrations on U87 and U251 cells. C) CCK-8 assay showed that the combination of curcumin and LIUS suppressed glioma cell viability in a synergistic manner. D) EdU assay further confirmed that curcumin plus LIUS significantly inhibited the proliferation of glioma cells. Scale bars $100 \mu \mathrm{m} .{ }^{* *} \mathrm{p}<0.01$ and ${ }^{* * *} \mathrm{p}<0.001$ vs. the control group. ${ }^{* * *} \mathrm{p}<0.01$ and ${ }^{* * * *} \mathrm{p}<0.001$ vs. the LIUS group. ${ }^{8} \mathrm{p}<0.05,{ }^{88} \mathrm{p}<0.01$, and ${ }^{8 \& 8} \mathrm{p}<0.001$ vs. the curcumin group. Assays were performed in triplicate and data are shown as mean $\pm \mathrm{SD}$. 
between the control group and the DMSO group, demonstrating that DMSO itself did not influence the viability of glioma cells. The cell viability rates were significantly reduced with increasing concentrations of curcumin, suggesting that higher concentrations of curcumin could be toxic to these cells (Figure 1B). These findings demonstrated that curcumin suppressed the growth of glioma cells in a dose-dependent manner, although practically no significant effect was found for curcumin concentrations below $10 \mu \mathrm{M}$.

Combination treatment of curcumin and LIUS synergistically reduces glioma cell viability. To explore the effect of combined treatment with LIUS and low-dose curcumin on the growth of glioma cells, CCK-8 and EdU assays were performed to determine the proliferative activity of glioma cells. As presented in Figures 1C, D, glioma cells were treated with LIUS $\left(83.4 \mathrm{~mW} / \mathrm{cm}^{2}\right)$, curcumin $(10 \mu \mathrm{M})$, and their combination for $24 \mathrm{~h}$. CCK- 8 assay showed that the cell viability rates of U87 cells in LIUS group, curcumin group, and curcumin + LIUS group were $94.8 \pm 6.2 \%, 91.5 \pm 8.2 \%$, and $70.9 \pm 2.4 \%$, respectively; the viability rates of U251 cells were $92.6 \pm 2.6 \%, 90.9 \pm 3.2 \%$, and $66.6 \pm 1.8 \%$, respectively (Figure 1C). Relative to the curcumin alone group, the inhibitory cell viability of the curcumin + LIUS group was 3.4-fold and 3.7-fold that of the curcumin group in U87 and U251 cells. Similarly, the EdU assay showed that the number of EdU-positive glioma cells in the curcumin + LIUS group was significantly reduced compared with that in the other three groups (Figure 1D). There was no significant difference among the control group, LIUS group, and curcumin group. These data indicated that LIUS in combination with curcumin synergistically reduced glioma cell proliferation.

Combination treatment of curcumin and LIUS synergistically promotes glioma cell apoptosis. Flow cytometry assay was carried out to determine whether LIUS plus curcumin could increase apoptosis in glioma cells. The apoptosis rate of U87 and U251 cells in the curcumin + LIUS group was $20.2 \pm 1.5 \%$ and $22.3 \pm 2.2 \%$, respectively, which was significantly higher than that of the control $(7.6 \pm 0.5 \%$ and $7.8 \pm 0.6 \%$, respectively), LIUS-alone $(8.3 \pm 0.6 \%$ and $9.5 \pm 1.0 \%$, respectively), and curcumin-alone groups $(9.7 \pm 0.8 \%$ and $10.8 \pm 1.2 \%$, respectively) (Figures $2 \mathrm{~A}, 2 \mathrm{~B})$. The protein expression of Bax and Bcl-2 in U87 and U251 cells was detected by western blot analysis, the results demonstrated that there was no significant difference in the protein expression of Bax and Bcl-2 in U87 and U251 cells among the control group, LIUS group, and curcumin group (Figures $3 \mathrm{~A}, 3 \mathrm{~B})$. The protein expression of $\mathrm{Bcl}-2$ in $\mathrm{U} 87$ and $\mathrm{U} 251$ cells was attenuated, and that of Bax was augmented in the curcumin + LIUS group relative to the other three groups. These results demonstrated that curcumin in combination with LIUS significantly increased glioma cell apoptosis.

Synergistic inhibition of the AKT pathway in glioma cells by combined treatment with curcumin and LIUS. In consideration of the crucial role of the AKT pathway in modulating cell survival in diverse types of tumors, we investigated whether LIUS in combination with curcumin inhibited the growth of glioma cells via inhibition of the AKT pathway. The results of western blot analysis showed that the ratio of phosphorylated AKT to total AKT was significantly decreased in LIUS and curcumin combination treatment contrasted to control, LIUS, and curcumin alone groups (Figures 3A, 3B). The above results indicated that the cytotoxicity of combined treatment with LIUS and curcumin may be associated with the inhibition of the AKT pathway.

Effects of the AKT pathway on glioma cell apoptosis and anti-proliferation induced by the combination of LIUS and curcumin. To ascertain that the anti-tumor effects induced by the combination of LIUS and curcumin occurred via inhibition of the AKT pathway, we used the AKT pathway activator SC79. EdU and CCK-8 assays revealed that inactivation of the AKT pathway strongly sensitized U87 and U251 cells toward the cytotoxicity of LIUS in combination with curcumin (Figures 4A, 4B). The inhibitory cell viability induced by LIUS and curcumin in combination was rescued
A
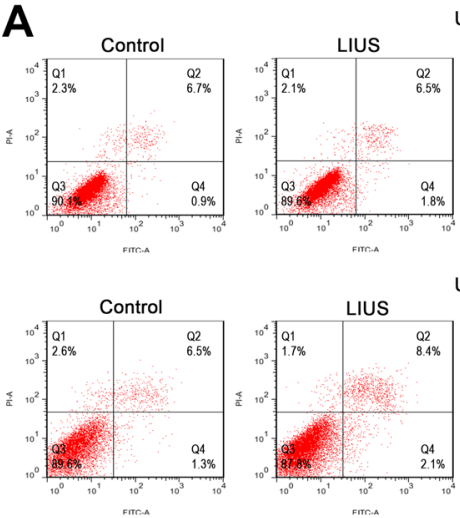

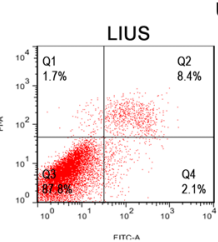

$87 \quad$ Curcumin

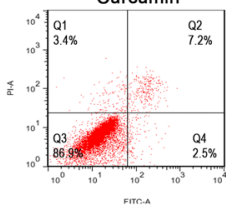

U251

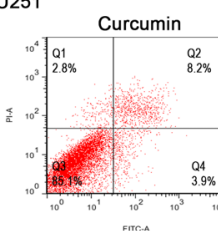

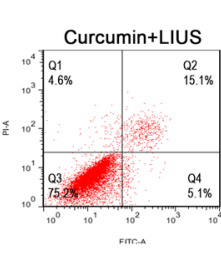

B

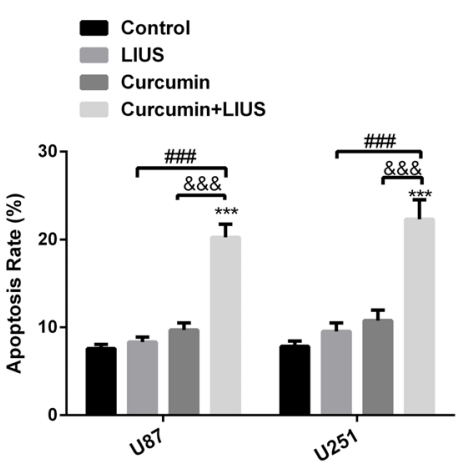

Figure 2. Apoptosis rate in U87 and U251 cells was detected by flow cytometry assay. A, B) The combination of LIUS and curcumin significantly promoted the apoptosis of glioma cells compared with the other three groups. ${ }^{* * *} \mathrm{p}<0.001$ vs. the control group. ${ }^{\# \#} \mathrm{p}<0.001$ vs. the LIUS group. ${ }^{\& \& \&} \mathrm{p}<0.001$ vs. the curcumin group. Assays were performed in triplicate and data are presented as mean \pm SD. 

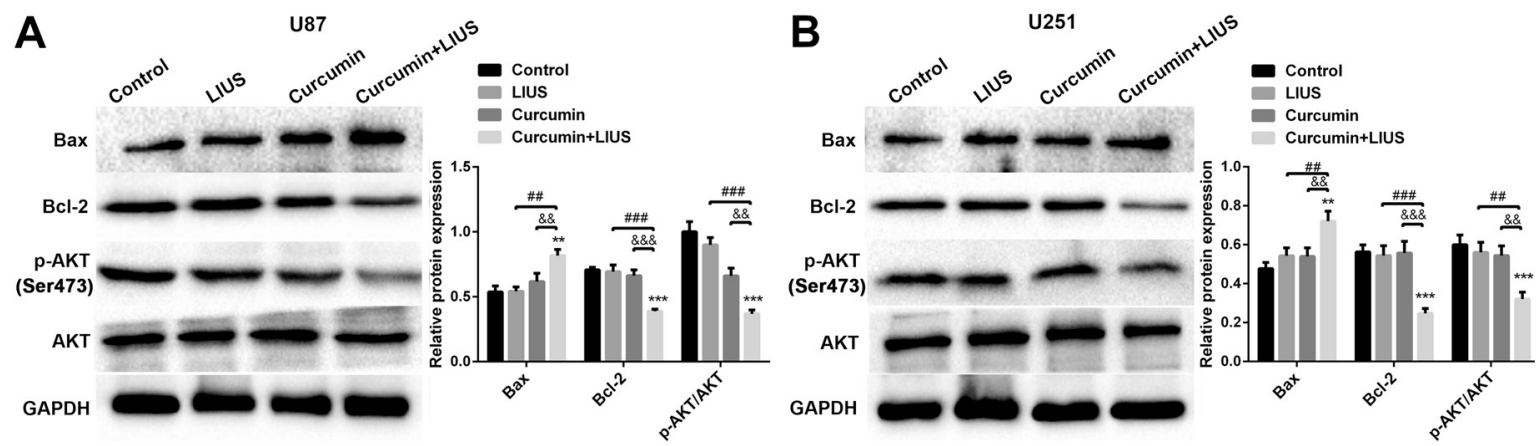

Figure 3. Levels of apoptosis-associated proteins and the proteins related to the AKT pathway in glioma cells treated with LIUS and curcumin. A, B) Western blot analysis showed that combined treatment with LIUS and curcumin significantly elevated Bax expression, while attenuated Bcl-2 level contrasted to the other three groups. Additionally, the ratio of p-AKT/AKT was decreased in the curcumin + LIUS group compared with that in the other three groups. ${ }^{* *} \mathrm{p}<0.01$ and ${ }^{* * *} \mathrm{p}<0.001$ vs. the control group. ${ }^{\# *} \mathrm{p}<0.01$ and ${ }^{\# \# *} \mathrm{p}<0.001$ vs. the LIUS group. ${ }^{\& \&} \mathrm{p}<0.01$ and ${ }^{\& \& \&} \mathrm{p}<0.001$ vs. the curcumin group. Assays were performed in triplicate and data are shown as mean \pm SD.

when treatment with SC79, suggesting that the AKT pathway is required for the inhibitory effects induced by LIUScurcumin combination.

Flow cytometry assay showed that cells co-treated with SC79 and curcumin +LIUS had a lower apoptotic rate than those with curcumin + LIUS treatment (U87: $8.0 \pm 0.9 \%$ vs. 20.3 $\pm 2.0 \%$; and U251: $7.6 \pm 0.7 \%$ vs. $24.0 \pm 1.9 \%$, Figure $4 \mathrm{C}$ ). As presented in Figure 4D, co-treatment with SC79 and curcumin + LIUS significantly attenuated Bax expression but augmented the levels of Bcl-2 and p-AKT/AKT, suggesting that the apoptosis process was suppressed when compared with the LIUS-curcumin combination. Hence, activation of the AKT pathway using SC79 treatment could reverse the promotion of cell apoptosis mediated by LIUS and curcumin in combination. The above results indicated an involvement of the AKT pathway in regulating both cell apoptosis and the anti-proliferation process caused by LIUS-curcumin combination.

\section{Discussion}

Within a certain range of ultrasonic intensity, cell proliferation decreases with the increase of ultrasonic intensity. When it exceeds the maximum range, cell structure and morphology might be changed or even damaged. In this study, the viability rates of glioma cells presented a decreased tendency with the increase of ultrasonic intensities. In contrast to the control group, there was no significant effect on glioma cell viability when the ultrasonic intensity was below $83.4 \mathrm{~mW} / \mathrm{cm}^{2}$. These findings reveal that LIUS individually inhibited the proliferation of glioma cells in an intensity-dependent manner.

Numerous studies on the anti-tumor activities and mechanisms of curcumin have been widely investigated both in vivo and in vitro. Wang et al. explored the anti-tumor mechanisms of curcumin in treating gastric cancer by downregulating the STAT3 signal pathway [20]. Curcumin exerted anti- tumor effects on diffuse large B cell lymphoma via regulating PPAR $\gamma$ expression [21]. In this study, compared with the control group, there was a significant difference in glioma cell viability with curcumin concentration greater than $10 \mu \mathrm{M}$. And with the increase of curcumin concentration, the cell viability significantly decreased, indicating that curcumin inhibited the proliferation of glioma cells in a dose-dependent manner.

As a promising technique, LIUS has been able to open the blood-brain barrier and promote the delivery of a series of therapeutic agents, including low-molecular-weight drugs and antibodies [22]. It is well reported that the targeted drug delivery has been observed by using the microbubble composing ultrasound contrast agents to effectively improve drug or gene delivery efficiency [23]. Accumulating literature has demonstrated that the mechanisms by which LIUS could increase the permeability of cell membrane may be due to the combination of mechanical and cavitation effects, thus enhancing the delivery of chemotherapeutic drugs [24, 25]. This evidence also demonstrates that LIUS can function as an assistant tool to deliver curcumin in the treatment of glioma. In the present study, the combination treatment of curcumin and LIUS significantly inhibited the growth of glioma cells in a synergistic manner. And the synergistic effect induced by curcumin and LIUS in combination, such as lower cell viability, more cell apoptosis, and lower level of p-AKT/ AKT, was much greater than that of every single treatment. Therefore, we speculate that the synergistic effect may be attributable to the LIUS-mediated increase in permeability of cell membrane, resulting in intracellular accumulation of curcumin, thus inhibiting proliferation but promoting apoptosis in glioma cells.

In this study, CCK-8 and EdU assays showed that the combination of curcumin and LIUS synergistically inhibited glioma cell proliferation. To illustrate the apoptosis induction by LIUS-curcumin combination, flow cytometry analysis was executed. And it was observed that LIUS combined with 

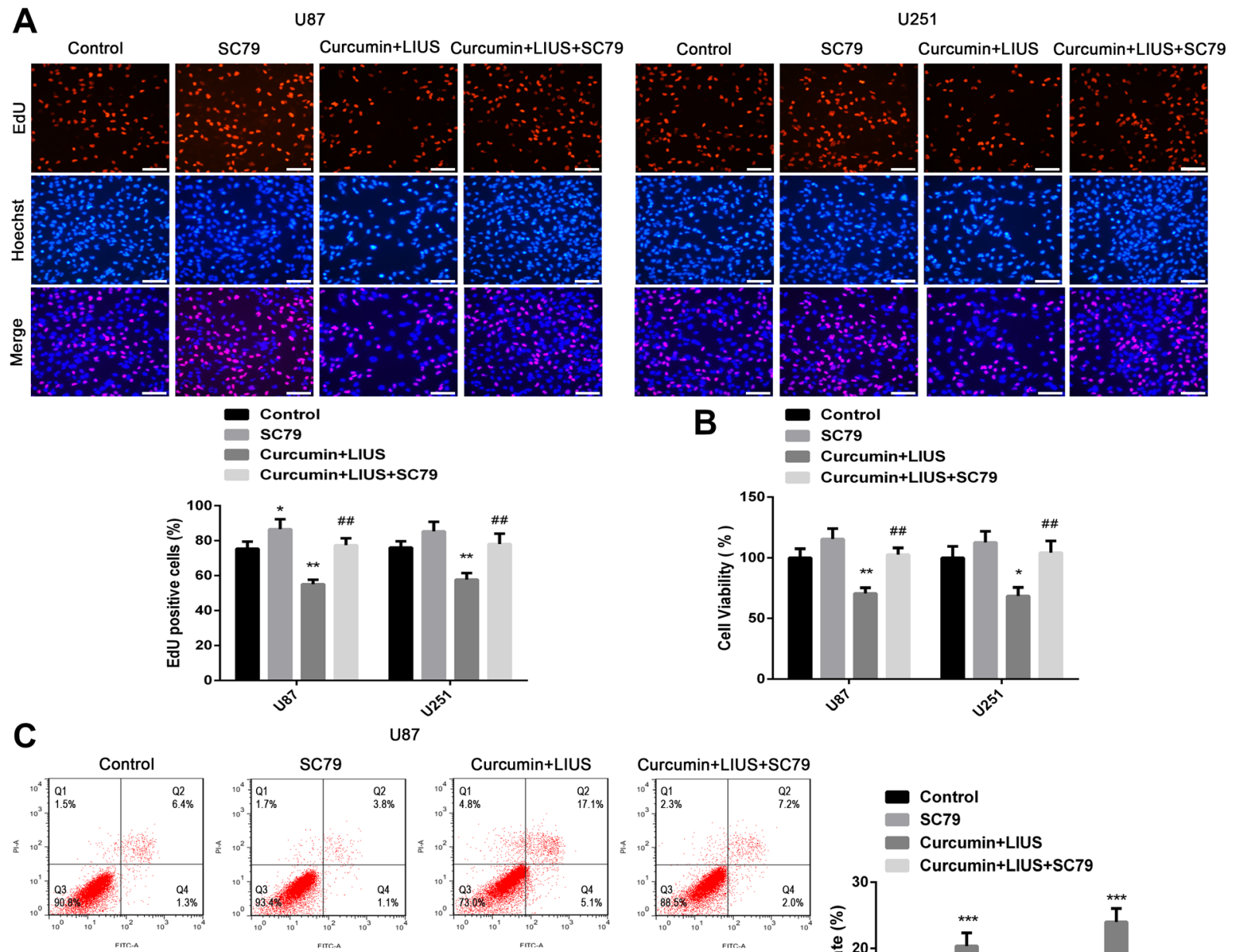

U87
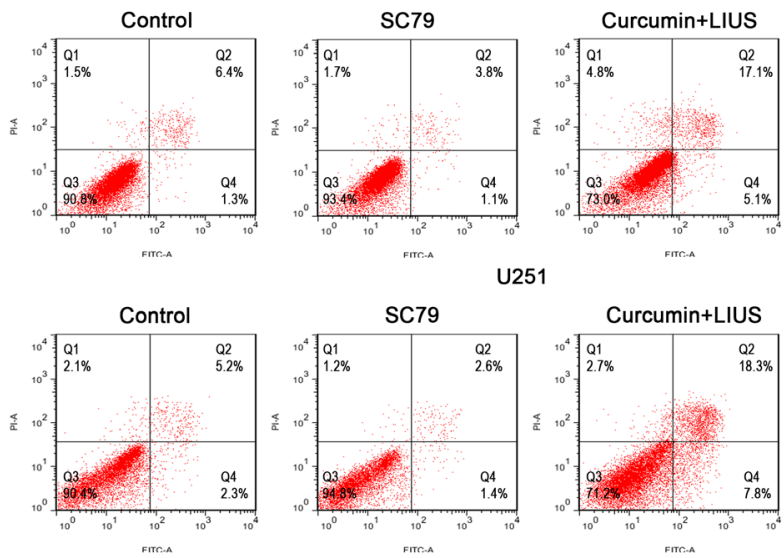

U251
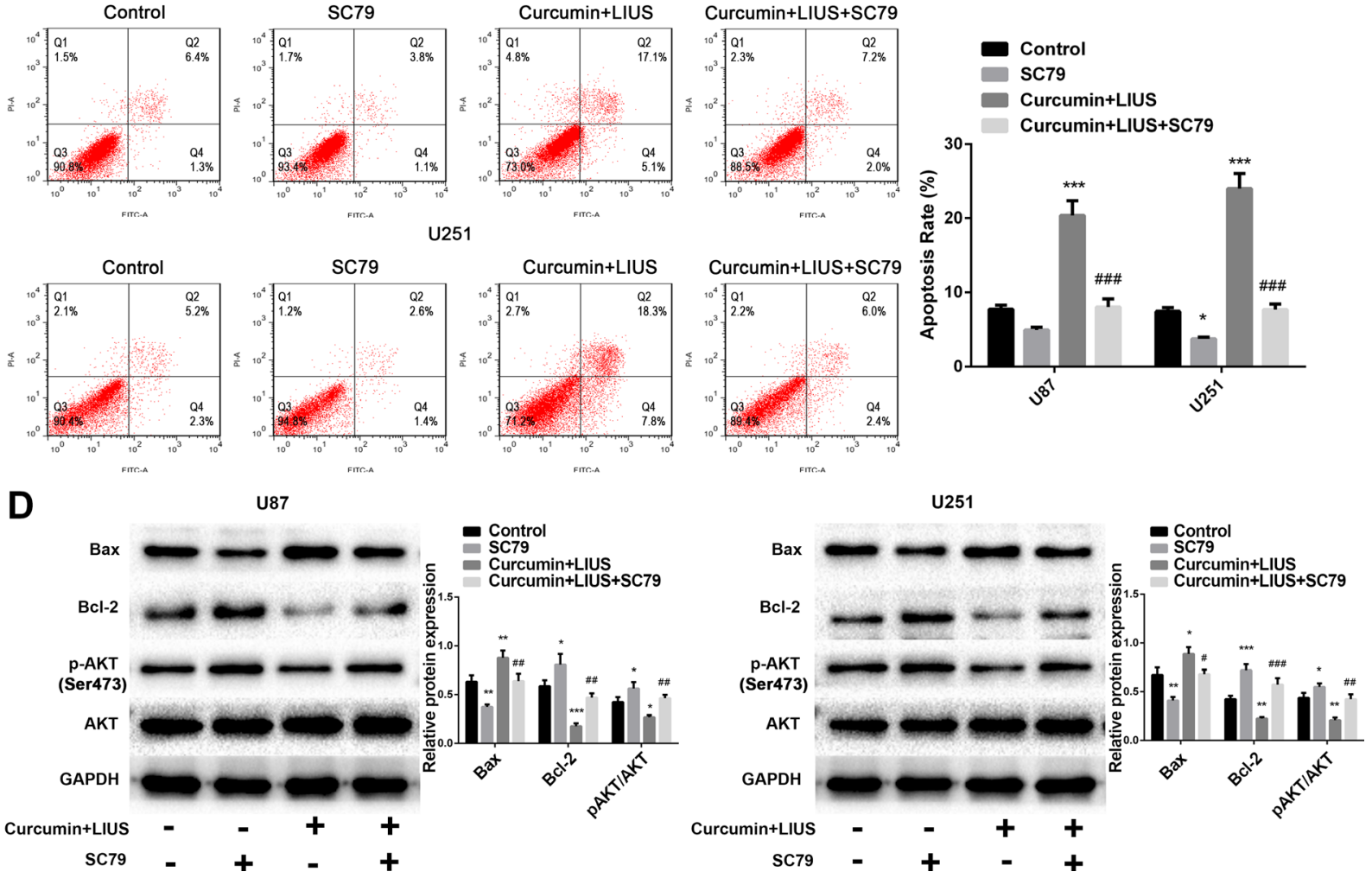

Figure 4. Effects of the AKT pathway on LIUS-curcumin combination-mediated cell anti-proliferation and apoptosis. U87 and U251 cells were treated with the LIUS-curcumin combination in the presence or absence of SC79. A, B) EdU and CCK-8 assays showed that treatment with SC79 rescued the reduced cell proliferation induced by LIUS and curcumin combination treatment. Scale bars $100 \mu \mathrm{m}$. C) Flow cytometry assay revealed the percentage of apoptotic cell populations following treatment with LIUS-curcumin combination in the presence or absence of SC79. D) The protein levels of Bax, Bcl-2, and p-AKT/AKT were detected by western blot analysis. ${ }^{*} \mathrm{p}<0.05,{ }^{* *} \mathrm{p}<0.01$, and ${ }^{* * *} \mathrm{p}<0.001$ vs. the control group. ${ }^{*} \mathrm{p}<0.05$, ${ }^{* *} \mathrm{p}<0.01$, and ${ }_{* \# \# * 0} \mathbf{p}<0.001$ vs. the curcumin + LIUS group. Assays were performed in triplicate and data are shown as mean \pm SD. 
curcumin elicited a significant increase in the apoptosis rate of glioma cells. Similarly, the results of western blot analysis showed that significant upregulation of Bax and downregulation of Bcl-2 protein were observed by the combined treatment with LIUS and curcumin in U87 and U251 cells, indicating that apoptosis was induced, which is consistent with the findings of other investigators, who reported that curcumin plus berberine led to dramatically elevated Bax and reduced $\mathrm{Bcl}-2$ expression in glioblastoma [17]. Several studies reported that Bax and Bcl-2 played a critical role in determining the ultimate fate of tumor cells, as indicated that Bcl-2 restrained apoptosis by stabilizing the mitochondrial membrane potential, while elevated Bax expression promoted cell apoptosis due to the release of cytochrome c $[26,27]$. These findings suggest that the LIUS-curcumin combination synergistically promotes anti-proliferation and apoptosis of glioma cells.

Several studies showed that curcumin restrained tumor cell proliferation and induced apoptosis through modulating the AKT/mTOR signaling $[28,29]$. PI3K/AKT is one of the most important signaling pathways, which is required for tumor cell survival [30]. The activated AKT pathway can make glioma cells avoid apoptosis and promote their growth. So downregulation of the AKT pathway may be an attractive target of chemotherapy against human glioma. Our previous research reported the reversal of multidrug resistance protein 1 (MRP1) expression caused by LIUS and curcumin via the VEGF/PI3K/AKT pathway in brain glioma [31]. Therefore, here we also investigated the role of the AKT pathway in the process of glioma cell apoptosis and anti-proliferation induced by the combination of LIUS and curcumin. The results of the western blot assay showed that the ratio of phosphorylated AKT to total AKT was significantly downregulated by the combination of LIUS and curcumin, thereby suggesting that LIUS plus curcumin blocked the AKT pathway in human glioma cells. Additionally, a novel AKT activator SC79 was employed to further determine the anti-proliferation and apoptosis induced by the LIUS-curcumin combination via inhibition of the AKT pathway. In this study, significant suppression of the AKT pathway occurred in the combined treatment with LIUS and curcumin, which could be alleviated by SC79 treatment. Also, treatment with activator SC79 reversed the anti-proliferation and apoptosis mediated by LIUS-curcumin combination in glioma cells. Briefly, the occurrence of anti-proliferation and apoptosis after LIUScurcumin combination treatment was attributed to the suppression of the AKT pathway, thus indicating that this pathway had a crucial role in the LIUS-curcumin combination treatment of glioma cells. These findings suggest that the AKT pathway could be involved in LIUS-curcumin mediated inhibition of glioma cell growth. The progression of cancer is an extremely complex process, associated with variable factors and characteristics between different types of this disease. Tumor cell survival or death should not be determined by only one signaling pathway. And xenograft experiments should be performed to confirm our findings in vivo. Further investigation on the association between LIUS plus curcumin-induced anti-proliferation and apoptosis as well as the detailed mechanisms in glioma cells represents the direction of our future research.

In conclusion, in this study LIUS $\left(83.4 \mathrm{~mW} / \mathrm{cm}^{2}\right)$ or curcumin $(10 \mu \mathrm{M})$ alone did not produce significant antitumor effects, but the combination of LIUS and curcumin synergistically inhibited the growth of glioma cells via inhibition of the AKT pathway. As a non-invasive potential therapeutic strategy, combined treatment with low-dose curcumin and LIUS represents an innovative approach that greatly reduces curcumin dosage to minimize side effects and warrants further investigation toward possible clinical application in patients with malignant glioma.

Acknowledgments: This research was supported by the Natural Science Foundation of Liaoning Province, China (2019-ZD0772) and the National Natural Science Foundation of China (No. 81471809; No. 81971639).

\section{References}

[1] ALIFIERIS C, TRAFALIS DT. Glioblastoma multiforme: Pathogenesis and treatment. Pharmacol Ther 2015; 152: 6382. https://doi.org/10.1016/j.pharmthera.2015.05.005

[2] STUPP R, HEGI ME, MASON WP, VAN DEN BENT MJ, TAPHOORN MJ et al. Effects of radiotherapy with concomitant and adjuvant temozolomide versus radiotherapy alone on survival in glioblastoma in a randomised phase III study: 5-year analysis of the EORTC-NCIC trial. Lancet Oncol 2009; 10: 459-466. https://doi.org/10.1016/S14702045(09)70025-7

[3] WANG X, DENG J, YUAN J, TANG X, WANG Y et al. Curcumin exerts its tumor suppressive function via inhibition of NEDD4 oncoprotein in glioma cancer cells. Int J Oncol 2017; 51: 467-477. https://doi.org/10.3892/ijo.2017.4037

[4] BIMONTE S, BARBIERI A, LEONGITO M, PICCIRILLO M, GIUDICE A et al. Curcumin AntiCancer Studies in Pancreatic Cancer. Nutrients 2016; 8: 433. https://doi. org/10.3390/nu8070433

[5] KASI PD, TAMILSELVAM R, SKALICKA-WOZNIAK K, NABAVI SF, DAGLIA $M$ et al. Molecular targets of curcumin for cancer therapy: an updated review. Tumour Biol 2016; 37: 13017-13028. https://doi.org/10.1007/s13277016-5183-y

[6] YAlLAPU MM, JAGGI M, CHAUHAN SC. Curcumin nanomedicine: a road to cancer therapeutics. Curr Pharm Des 2013; 19: 1994-2010. https://doi. org/10.2174/138161213805289219

[7] ZHANG Z, CHEN J, CHEN L, YANG X, ZHONG H et al. Low frequency and intensity ultrasound induces apoptosis of brain glioma in rats mediated by caspase-3, Bcl-2, and survivin. Brain Res 2012; 1473: 25-34. https://doi.org/10.1016/j. brainres.2012.06.047 
[8] SHEIKOV N, MCDANNOLD N, VYKHODTSEVA N, JOLESZ F, HYNYNEN K. Cellular mechanisms of the bloodbrain barrier opening induced by ultrasound in presence of microbubbles. Ultrasound Med Biol 2004; 30: 979-989. https://doi.org/10.1016/j.ultrasmedbio.2004.04.010

[9] SHEIKOV N, MCDANNOLD N, SHARMA S, HYNYNEN K. Effect of focused ultrasound applied with an ultrasound contrast agent on the tight junctional integrity of the brain microvascular endothelium. Ultrasound Med Biol 2008; 34: 10931104. https://doi.org/10.1016/j.ultrasmedbio.2007.12.015

[10] NITULESCU GM, VAN DE VENTER M, NITULESCU G, UNGURIANU A, JUZENAS P et al. The Akt pathway in oncology therapy and beyond (Review). Int J Oncol 2018; 53: 2319-2331. https://doi.org/10.3892/ijo.2018.4597

[11] GUANEN Q, JUNJIE S, BAOLIN W, CHAOYANG W, YAJUAN Y et al. MiR-214 promotes cell meastasis and inhibites apoptosis of esophageal squamous cell carcinoma via PI3K/AKT/mTOR signaling pathway. Biomed Pharmacother 2018; 105: 350-361. https://doi.org/10.1016/j.biopha.2018.05.149

[12] ZHAO H, CHEN G, YE L, YU H, LI S et al. DOK7V1 influences the malignant phenotype of lung cancer cells through $\mathrm{PI} 3 \mathrm{~K} / \mathrm{AKT} / \mathrm{mTOR}$ and FAK/paxillin signaling pathways. Int J Oncol 2019; 54: 381-389. https://doi.org/10.3892/ ijo.2018.4624

[13] ZHAO J, LI Z, CHEN Y, ZHANG S, GUO L et al. MicroRNA766 inhibits papillary thyroid cancer progression by directly targeting insulin receptor substrate 2 and regulating the PI3K/Akt pathway. Int J Oncol 2019; 54: 315-325. https://doi.org/10.3892/ijo.2018.4615

[14] LIU SQ, XU CY, WU WH, FU ZH, HE SW et al. Sphingosine kinase 1 promotes the metastasis of colorectal cancer by inducing the epithelialmesenchymal transition mediated by the FAK/AKT/MMPs axis. Int J Oncol 2019; 54: 41-52. https://doi.org/10.3892/ijo.2018.4607

[15] KOUNDOUROS N, POULOGIANNIS G. Phosphoinositide 3-Kinase/Akt Signaling and Redox Metabolism in Cancer. Front Oncol 2018; 8: 160. https://doi.org/10.3389/ fonc. 2018.00160

[16] MCDOWELL KA, RIGGINS GJ, GALLIA GL. Targeting the AKT pathway in glioblastoma. Curr Pharm Des 2011; 17: 2411-2420. https://doi.org/10.2174/138161211797249224

[17] MAITI P, PLEMMONS A, DUNBAR GL. Combination treatment of berberine and solid lipid curcumin particles increased cell death and inhibited PI3K/Akt/mTOR pathway of human cultured glioblastoma cells more effectively than did individual treatments. PLoS One 2019; 14: e0225660. https:// doi.org/10.1371/journal.pone.0225660

[18] MAITI P, SCOTT J, SENGUPTA D, AL-GHARAIBEH A, DUNBAR GL. Curcumin and Solid Lipid Curcumin Particles Induce Autophagy, but Inhibit Mitophagy and the PI3KAkt/mTOR Pathway in Cultured Glioblastoma Cells. Int J Mol Sci 2019; 20. https://doi.org/10.3390/ijms20020399

[19] LIN HY, THOMAS JL, CHEN HW, SHEN CM, YANG WJ et al. In vitro suppression of oral squamous cell carcinoma growth by ultrasound-mediated delivery of curcumin microemulsions. Int J Nanomedicine 2012; 7: 941-951. https://doi. org/10.2147/IJN.S28510
[20] WANG XP, WANG QX, LIN HP, CHANG N. Anti-tumor bioactivities of curcumin on mice loaded with gastric carcinoma. Food Funct 2017; 8: 3319-3326. https://doi. org/10.1039/c7fo00555e

[21] ZHANG W, LI Q, YANG C, YANG H, RAO J, et al. Curcumin exerts anti-tumor effects on diffuse large B cell lymphoma via regulating PPARgamma expression. Biochem Biophys Res Commun 2020; 524: 70-76. https://doi.org/10.1016/j. bbrc.2019.12.129

[22] BECCARIA K, CANNEY M, BOUCHOUX G, PUGET S, GRILL J et al. Blood-brain barrier disruption with low-intensity pulsed ultrasound for the treatment of pediatric brain tumors: a review and perspectives. Neurosurg Focus 2020; 48: E10. https://doi.org/10.3171/2019.10.FOCUS19726

[23] BOUAKAZ A, ZEGHIMI A, DOINIKOV AA. Sonoporation: Concept and Mechanisms. Adv Exp Med Biol 2016; 880: 175-189. https://doi.org/10.1007/978-3-319-22536$4 \_10$

[24] HU Z, LV G, LI Y, LI E, LI H et al. Enhancement of antitumor effects of 5-fluorouracil on hepatocellular carcinoma by low-intensity ultrasound. J Exp Clin Cancer Res 2016; 35: 71. https://doi.org/10.1186/s13046-016-0349-4

[25] LAMMERTINK B, DECKERS R, STORM G, MOONEN C, BOS C. Duration of ultrasound-mediated enhanced plasma membrane permeability. Int J Pharm 2015; 482: 92-98. https://doi.org/10.1016/j.ijpharm.2014.12.013

[26] HE W, LI X, XIA S. Lupeol triterpene exhibits potent antitumor effects in A427 human lung carcinoma cells via mitochondrial mediated apoptosis, ROS generation, loss of mitochondrial membrane potential and downregulation of m-TOR/PI3Ksol;AKT signalling pathway. J BUON 2018; 23: 635-640

[27] LUNA ACL, SARAIVA GKV, CHIERICE GO, HESSE H, MARIA DA. Antiproliferative and proapoptotic effects of DODAC/synthetic phosphoethanolamine on hepatocellular carcinoma cells. BMC Pharmacol Toxicol 2018; 19: 44. https://doi.org/10.1186/s40360-018-0225-2

[28] ZHAO G, HAN X, ZHENG S, LI Z, SHA Y et al. Curcumin induces autophagy, inhibits proliferation and invasion by downregulating AKT/mTOR signaling pathway in human melanoma cells. Oncol Rep 2016; 35: 1065-1074. https://doi. org/10.3892/or.2015.4413

[29] HU S, XU Y, MENG L, HUANG L, SUN H. Curcumin inhibits proliferation and promotes apoptosis of breast cancer cells. Exp Ther Med 2018; 16: 1266-1272. https://doi. org/10.3892/etm.2018.6345

[30] SHARMA VR, GUPTA GK, SHARMA AK, BATRA N, SHARMA DK, et al. PI3K/Akt/mTOR Intracellular Pathway and Breast Cancer: Factors, Mechanism and Regulation. Curr Pharm Des 2017; 23: 1633-1638. https://doi.org/10.217 4/1381612823666161116125218

[31] YAO L, ZHANG Z. The reversal of MRP1 expression induced by low-frequency and low-intensity ultrasound and curcumin mediated by VEGF in brain glioma. Onco Targets Ther 2019; 12: 3581-3593. https://doi.org/10.2147/OTT. S195205 